\title{
Research Challenges for Zero Carbon and Sustainable Fuels: Prospects and the Pathway Forward
}

\author{
M. Pourkashanian \\ Energy Institute, University of Sheffield, \\ Sheffield, S102TN, UK \\ Address for corresponding: m.pourkashanian@sheffield.ac.uk
}

\begin{abstract}
The world drive to reach net zero by 2050 will require some big changes, including the need for the total transformation of our energy systems. So how can we get to the Net Zero target by 2050 ? What will be the role of translational and innovative energy technologies? Whilst there are many options and pathways that could in theory get us to this target, understanding the 'best' approach is something that is open to clarification.

A new report by the IEA highlights pathways to the Net Zero target that might be possible through implementation of innovative technology and scale-up. The pathways reflect consideration of a whole range of implementation approaches and highlight the consequences of differing levels of technological innovation and societal behaviour changes. Key Low Carbon Technology options include carbon capture, use and storage; hydrogen and zero carbon fuels for heat and industrial decarbonisation, bioenergy; energy efficiency, electrification and renewables.

In this paper, the role research and innovation can play in delivering the NetZero target was assessed by examining two cases from fundamental research into pilot scale in order to accelerate innovation. It is clear that rapid innovation has a key role to play and targeting on technologies that have the greatest potential to slash carbon emissions. The research reported here includes the next generation carbon capture technologies. Rotating packed beds (RPB) could have a significant impact on the capture cost and efficiency of the process. RPBs are a type of process intensification technology that have been proposed as an emerging technology to be used for post combustion capture from the flue gas. This is because of its high mass transfer coefficient and compact structure which will lead to substantial energy and space savings, hence significantly lowering the cost of the $\mathrm{CO} 2$ capture. The first case investigated examines the process from fundamental research into large scale testing as a part of the commercialisation. Finally, in the second case, the impact of a breakthrough technology, a supercritical $\mathrm{CO} 2$ (sCO2) based energy system is examined and the research challenges are assessed. The sCO2 technology has the potential to produce electricity at a lower cost than conventional fossil fuel generation - with the inherent carbon capture and zero air emissions. However, there are concerns with thermal management and the temperatures inside the turbine. The simulations of natural gas combustion that has only been validated for low pressures and without heavy dilution of $\mathrm{CO} 2$ is an important tool to identify the design challenges. The development of a robust research scheme for high pressure and high temperature is explained in this paper.
\end{abstract}

